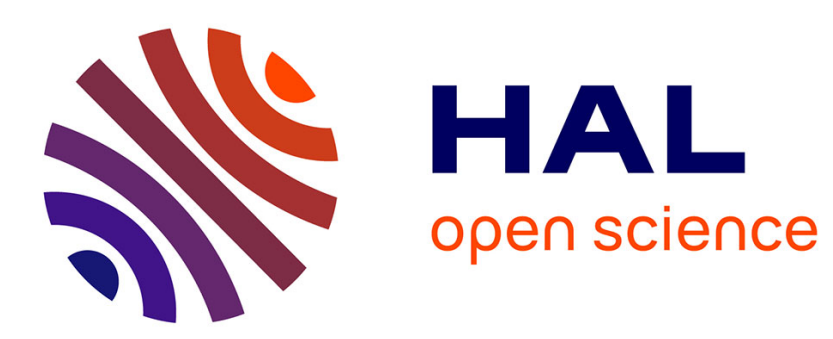

\title{
Le droit de vote des étrangers: une utopie déjà réalisée sur les cinq continents
}

\author{
Hervé Andres
}

\section{To cite this version:}

Hervé Andres. Le droit de vote des étrangers: une utopie déjà réalisée sur les cinq continents. Migrations Société, 2007, 19 (114), pp.65-81. halshs-00189681v2

\section{HAL Id: halshs-00189681 https://shs.hal.science/halshs-00189681v2}

Submitted on 19 Jun 2017

HAL is a multi-disciplinary open access archive for the deposit and dissemination of scientific research documents, whether they are published or not. The documents may come from teaching and research institutions in France or abroad, or from public or private research centers.
L'archive ouverte pluridisciplinaire HAL, est destinée au dépôt et à la diffusion de documents scientifiques de niveau recherche, publiés ou non, émanant des établissements d'enseignement et de recherche français ou étrangers, des laboratoires publics ou privés. 


\section{LE DROIT DE VOTE DES ÉTRANGERS : UNE UTOPIE DÉJÀ RÉALISÉE SUR LES CINQ CONTINENTS}

Hervé Andrès

Centre d'information et d'études sur les migrations internationales | «Migrations Société »

$2007 / 6 N^{\circ} 114 \mid$ pages 65 à 81

ISSN 0995-7367

Article disponible en ligne à l'adresse :

http://www.cairn.info/revue-migrations-societe-2007-6-page-65.htm

\section{Pour citer cet article :}

Hervé Andrès, « Le droit de vote des étrangers : une utopie déjà réalisée sur les cinq continents », Migrations Société 2007/6 (N¹14), p. 65-81.

Distribution électronique Cairn.info pour Centre d'information et d'études sur les migrations internationales.

(C) Centre d'information et d'études sur les migrations internationales. Tous droits réservés pour tous pays.

La reproduction ou représentation de cet article, notamment par photocopie, n'est autorisée que dans les limites des conditions générales d'utilisation du site ou, le cas échéant, des conditions générales de la licence souscrite par votre établissement. Toute autre reproduction ou représentation, en tout ou partie, sous quelque forme et de quelque manière que ce soit, est interdite sauf accord préalable et écrit de l'éditeur, en dehors des cas prévus par la législation en vigueur en France. Il est précisé que son stockage dans une base de données est également interdit. 


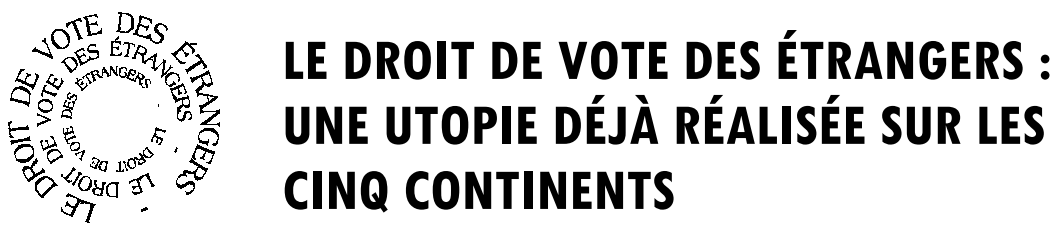

Hervé ANDRÈS *

II y a une douzaine d'années, Francis Delpérée expliquait que pendant longtemps la question des droits politiques des étrangers n'aurait pas retenu la moindre attention et que la règle d'exclusion de l'étranger de la vie politique prévalait encore très largement, y compris en Europe ${ }^{1}$. La participation des étrangers à des scrutins était exceptionnelle. Comme l'indiquait Danièle Lochak, l'étranger apparaissait universellement comme l'éternel exclu et, en matière politique, le système des États-nations avait consolidé l'irréductible frontière entre le national et l'étranger, privé des droits politiques en général et du droit de vote en particulier ${ }^{2}$.

Le droit de vote des étrangers constitue indéniablement un des problèmes de fond pour la démocratie à l'aube du XXIe siècle. II est un point de friction où démocratie et souveraineté entrent en contradiction $^{3}$. Ce problème se pose, potentiellement, partout sur la planète, dans le cadre des États souverains. La citoyenneté semble totalement absorbée par la nationalité, c'est-à-dire par la souveraineté ${ }^{4}$. La situation européenne est assez connue et largement utilisée en France dans le débat sur le droit de vote des étrangers, à la fois parce que les différentes expériences des pays européens en la matière, à partir des années 70, ont inspiré les pays voisins, et aussi parce que l'intégration croissante des États dans l'Union européenne a entraîné la rupture du modèle théorique entre nationalité et citoyenneté,

* Ingénieur d'études, docteur en science politique, Université de Nice, Unité de recherches Migrations et société (URMIS), CNRS, UMR 7032. E-mail : herve.andres@unice.fr

1. Cf. DELPÉRÉE, Francis, Les droits politiques des étrangers, Paris : Presses universitaires de France, 1995, collection "Que sais-je ?", $127 \mathrm{p}$

2. Voir LOCHAK, Danièle, Étrangers : de quel droit ?, Paris : Presses universitaires de France, 1985, chapitre "Les stigmates de l'infériorité", partie "Homo politicus", pp. 130-136.

3. Voir, dans ce même numéro, notre contribution sur les enjeux théoriques du droit de vote des étrangers, p. 47.

4. Cf. CHEMILLIER-GENDREAU, Monique, "Quelle citoyenneté universelle adaptée à la pluralité du monde ?", in : DAYAN-HERZBRUN, Sonia ; TASSIN, Etienne (sous la direction de), Citoyennetés cosmopolitiques, Tumultes, $\mathrm{n}^{\circ}$ 24, mai 2005, pp. 165-178. 
notamment par l'instauration du droit de vote lors des élections municipales et européennes pour les "citoyens de l'Union".

Mais quelle est la situation du droit de vote des étrangers hors d'Europe? La règle générale, qui est celle de l'exclusion des étrangers des droits politiques, est-elle confirmée partout, dans les faits et dans les textes? L'objet de cette contribution est de faire le point sur cette question, de manière relativement descriptive, en dressant un bref tableau, assez complet ${ }^{6}$, bien que non exhaustif, de la situation sur les autres continents ${ }^{7}$. L'intérêt est de montrer que - dans la diversité des réalités politiques, économiques, culturelles, géographiques et historiques - la question du droit de vote des étrangers est partout posée, et que, contrairement aux idées reçues, la pratique du vote des étrangers n'est pas du tout limitée à l'Europe. Sur les cinq continents, des étrangers ont le droit de vote, et l'on trouve au moins 35 pays non européens qui connaissent cette pratique. De plus, le droit de vote des étrangers n'est pas seulement une proposition du tournant du XXle siècle. Certains pays l'ont pratiqué durant de longues décennies, depuis les XVIIIe et XIXe siècles.

\section{En Amérique du Nord et en Amérique centrale, une pratique ancienne et répandue}

Pour dresser un tableau de la situation en Amérique du Nord et en Amérique centrale ${ }^{8}$, il convient de distinguer deux cas particuliers : les États-Unis d'une part, et les États du Commonwealth d'autre part.

5. Voir, dans ce même numéro, la contribution de Paul Oriol sur le droit de vote des résidents étrangers dans l'Union européenne, p. 83.

6. La littérature en la matière reste le plus souvent très incomplète, limitant l'étude hors d'Europe aux vieux pays d'immigration européenne.

7. On se limite aussi aux données concernant le droit de vote, sachant que la question de l'éligibilité des étrangers est également posée, dans des termes proches mais pas tout à fait identiques à ceux de la question du vote des étrangers. Par ailleurs, la mise en tableau de la situation du droit positif en la matière ne dispense pas d'une analyse critique prenant en compte la diversité des réalités historiques, économiques, démographiques, économiques, culturelles, géographiques, juridiques et politiques. Les données présentées ici ont pour la plupart été collectées en 2006, dans le cadre de la préparation d'une thèse sur cette question. Voir ANDRĖS, Hervé, Le droit de vote des étrangers : état des lieux et fondements théoriques, thèse de doctorat de sciences juridiques et politiques, spécialité de philosophie politique, sous la direction de $\mathrm{M}^{\mathrm{me}}$ Chemillier-Gendreau, Université Paris VII-Denis Diderot, École doctorale 382 - EESC Économie, Espaces, Sociétés, Civilisations : Pensée critique, politique et pratiques sociales, 2007, consultable en ligne à l'adresse suivante : http://tel.archives-ouvertes.fr/tel-00130445

8. Les données présentées ici sont principalement basées sur les sources trouvées en mai 2006 sur la base de données politiques des Amériques (Center for Latin American Studies, Georgetown University, http://pdba.georgetown.edu). Ce site collaboratif donne accès entre autres aux textes du droit positif (Constitutions, codes électoraux) en vigueur dans les différents États d'Amérique. 
La plupart des autres pays de cette région restreignent le droit de vote à leurs seuls ressortissants.

\section{- Aux États-Unis : une pratique longuement enracinée, oubliée, puis aujourd'hui redécouverte}

Aux États-Unis, la pratique du droit de vote des étrangers est très ancienne et a été très répandue. Entre 1776 et 1926, au moins 40 États ou territoires l'ont expérimentée 9 . Aujourd'hui, quelques communes accordent le droit de vote aux résidents étrangers, sous certaines conditions et pour certains types de scrutins ${ }^{10}$. Le débat a été relancé à la fin du $X X e$ siècle, dans le cadre du débat plus général sur la place de l'immigration dans la société étasunienne. Une particularité notable du débat étasunien est qu'il ne porte pas sur une mesure inédite, sans précédents, sur une utopie, mais au contraire sur le rétablissement d'une pratique ancienne, mais oubliée. II a fallu la conjonction de différents travaux universitaires, de mouvements sociaux et l'influence des évolutions en la matière sur tous les continents pour que la question émerge de nouveau dans le débat public aux États-Unis.

Les raisons pour lesquelles le droit de vote a été accordé aux étrangers durant l'histoire relèvent du mélange d'un pragmatisme utilitariste (consistant globalement à favoriser l'immigration) et des principes démocratiques ("No taxation without representation "), " No governance without representation "), etc.). C'est en tant que contribuables, et en tant que gouvernés, que les étrangers devaient être considérés comme des citoyens à part entière.

Une des formules à retenir, dans la longue histoire contrastée du droit de vote des étrangers aux États-Unis, est celle des "déclarants", mise au point par le Wisconsin lors de son admission à la fédération (1848). La Constitution du Wisconsin accordait le droit de vote aux étrangers ayant déclaré leur "intention" de devenir des citizens" ${ }^{11}$. Cette solution a été utilisée par de nombreux États, soucieux notamment d'encourager l'immigration européenne. Le droit de vote était ainsi vu comme un "chemin" vers la nationalité. En votant, les noncitizens devenaient des

9. Cf. HAYDUK, Ronald, Democracy for all : restoring immigrant voting rights in the United States, New York : Routledge Publisher, 2006, $250 \mathrm{p}$.

10. Voir le détail dans ANDRÈS, Hervé, Le droit de vote des étrangers, op. cit., chapitre 3.4, pp. 261-302.

11. Cf. ROSBERG, Gerald M., "Aliens and equal protection : why not the right to vote ?", Michigan Law Review, vol. 75, n 5-6, April-May 1977, pp. 1092-1136 (voir p. 1098). 
precitizens $^{12}$. II n'y avait en général pas de distinction entre droit de vote au niveau local et droit de vote au niveau de l'État ou au niveau fédéral. Cette solution a été critiquée car elle ne requérait pas l'allégeance aux États-Unis, elle rendait possible la double allégeance, et elle n'obligeait pas vraiment les "déclarants" à devenir citizens ${ }^{13}$.

Dans le conflit menant à la guerre de Sécession, les États du Sud, esclavagistes, ont récusé le droit de vote des étrangers au motif des convictions anti-esclavagistes supposées des immigrés. II est vrai que le Nord, anti-esclavagiste, avait mobilisé de nombreux étrangers. Du coup, et alors qu'elle exprimait une défiance vis-à-vis du pouvoir central et l'affirmation exacerbée du système fédéraliste, la Constitution sudiste (1863) interdit explicitement le droit de vote des étrangers ${ }^{14}$. Après la guerre civile, les États du Sud adoptèrent en général la formule du Wisconsin, accordant le droit de vote aux "déclarants", cherchant ainsi à aspirer de la main-d'œuvre pour remplacer les esclaves.

Le droit de vote des étrangers a été remis en question par les flambées de nationalisme suivant les périodes de guerre. C'est le patriotisme étasunien consécutif à la Première Guerre mondiale qui a sonné le glas (pour au moins une cinquantaine d'années) de cette pratique.

En 1931, Leon E. Aylsworth écrit un bref article qui signale la disparition " définitive " de cette pratique, qu'il qualifie d'u anomalie politique " : "Pour la première fois depuis plus de cent ans, une élection nationale s'est tenue en 1928 dans laquelle aucun étranger dans aucun État n'avait le droit de vote $11^{15}$. II explique que la tendance à accorder le droit de vote à des étrangers a connu son apogée vers 1875. II présente les différents processus, et notamment les référendums, qui ont conduit de nombreux États à supprimer ces dispositions jugées anormales. Son propos n'est pas de discuter sur le fond, qui semble indiscutable, mais plutôt de rendre compte des problèmes de procédures pour arriver à supprimer cette pratique qui lui paraît totalement illégitime. Les données historiques qu'il présente servent de base à la plupart des travaux menés sur la question jusqu'en 2005.

12. Cf. RASKIN, Jamin B., "Legal aliens, local citizens : the historical, constitutional, and theoretical meanings of alien suffrage", University of Pennsylvania Law Review, vol. 141, n 4, April 1993, pp. 1391-1470 (voir p. 1407).

13. Ibidem, p. 1406.

14. Ibidem, p. 1414.

15. AYLSWORTH, Leon E., "The passing of alien suffrage", The American Political Science Review vol. 25, n 1, February 1931, pp. 114-116. (cf. p.114). 
En 1977, Gerald M. Rosberg, professeur de droit à l'Université du Michigan, ouvre à nouveau la question, en s'étonnant que l'histoire du suffrage aux États-Unis, et en particulier de la pratique du vote des étrangers, ait été si peu écrite ${ }^{16}$. Discutant les fondements constitutionnels du suffrage, il remet en question l'idée selon laquelle le vote est par essence un privilège du citizen, et il montre que la jurisprudence abondante en la matière non seulement écarte l'exclusion des étrangers du droit de vote, mais tend même plutôt à justifier leur inclusion, au nom de la "clause d'égale protection", à tous les niveaux du gouvernement, contrairement à ce qui était communément admis.

En 1993, Jamin Raskin, professeur de droit constitutionnel (American University Washington College of Law) et protagoniste, l'année précédente, du rétablissement du droit de vote des étrangers dans sa ville de Takoma Park, près de Washington DC, publie un important article, qui constitue une ressource scientifique de référence et contribue à déclencher un nouveau mouvement pour les droits civiques, tout d'abord à San Francisco et à New York City ${ }^{17}$. Dans cet article, J. Raskin montre que le droit de vote des étrangers a joué un rôle historique extrêmement important dans la constitution des États-Unis. II ne s'agit pas du tout d'une pratique isolée, marquée par l'exceptionnalité et ayant une portée marginale. Au contraire, le droit de vote des étrangers a été longuement pratiqué dans de très nombreux États, et il a été débattu, contesté, supprimé, rétabli. II a donc été au centre du questionnement sur la nature de la politique étasunienne pendant plus de 150 ans.

Jamin Raskin montre aussi que la question du droit de vote des étrangers en général relève non pas de l'État fédéral et des principes constitutionnels, mais des États fédérés. Si la jurisprudence lui semble plaider pour une telle extension, il récuse la thèse de Gerald Rosberg (fondant la justification du droit de vote des étrangers sur la "clause d'égale protection") en signalant que ce n'est pas aux tribunaux que revient le pouvoir d'inclure dans l'électorat de nouvelles catégories jusque-là exclues, mais que ce pouvoir revient à l'électorat déjà constitué, qui finit toujours par entendre les revendications des aspirants-électeurs ${ }^{18}$.

16. Cf. ROSBERG, Gerald M., "Aliens and equal protection”, art. cité, p. 1093.

17. Cf. RASKIN, Jamin B., "Legal aliens, local citizens", art. cité.

18. Ibidem, partie II-D, "Is Alien Suffrage Constitutionaly Mandatory ?", pp. 1431-1441. 
Il présente enfin l'expérience de sa ville, Takoma Park, qui a accordé en 1992 le droit de vote lors des élections municipales à tous les résidents, instaurant ainsi une pratique déjà menée dans quelques communes du Maryland. II montre aussi que les expériences menées depuis quelques années à New York et à Chicago - où les étrangers parents d'élèves ont le droit de vote aux School board elections (élections politiques beaucoup plus importantes que leurs équivalentes en France) depuis la fin des années 60 - sont susceptibles d'être généralisées et ouvrent la voie à un mouvement général pour le droit de vote des étrangers au XXle siècle.

Effectivement, les années 90 ont connu différents mouvements, dans lesquels de nombreuses organisations se sont mobilisées pour obtenir le droit de vote des étrangers. Ces initiatives se sont insérées dans le cadre d'un mouvement plus large pour la reconnaissance des droits des immigrés et ont connu certains succès limités grâce au soutien actif d'élus importants. À l'heure où sont écrites ces lignes, quelques communes étasuniennes ont accordé le droit de vote aux étrangers, selon certaines conditions et à certains niveaux ${ }^{19}$.

L'histoire étasunienne du vote des étrangers, redécouverte aujourd'hui, se continue donc, notamment grâce à l'apport d'universitaires engagés dans la recherche sur ce sujet. Un des plus actifs désormais, outre Jamin Raskin, est Ronald Hayduk, professeur de science politique (Borough of Manhattan Community College, City University of New York), qui a produit de nombreux articles et ouvrages ${ }^{20}$. Il a dirigé une enquête sur l'histoire de cette pratique aux États-Unis et il s'est aussi engagé fortement dans le mouvement à New York et aux États-Unis. Il codirige avec l'universitaire Michele Wucker le site internet www.immigrantvoting.org, qui regroupe les données les plus pertinentes non seulement sur l'histoire du vote des étrangers aux États-Unis, mais aussi sur les développements actuels.

Si le contexte historique est totalement différent de celui de l'Europe, le débat sur le droit de vote des étrangers aux États-Unis présente tout de même de nombreux points communs. II s'agit, au fond, d'un débat sur l'essence de la citoyenneté, sur la réalité de la démocratie. Les partisans du droit de vote des étrangers, encore largement minoritaires, s'inscrivent dans la perspective de renforcer la démocratie, par l'ouverture et l'inclusion la plus universelle possible dans la sphère

19. Essentiellement dans l'État du Maryland.

20. Voir sa bibliographie sur le site http://www.ronhayduk.com. 
politique. Ils privilégient la dimension de la participation, postulant que c'est l'action politique (et notamment, en particulier, l'acte précis, symbolique et instrumental du vote) qui crée des citoyens. S'appuyant sur la longue histoire du droit de vote des étrangers, ils situent la revendication actuelle dans la tradition révolutionnaire universaliste de leur pays. À l'opposé, l'on objecte que le droit de vote doit rester une prérogative de la nationalité, marquant l'allégeance à l'État souverain et l'adhésion au pacte collectif de la nation étasunienne ${ }^{21}$.

\section{- Le droit de vote pour les "citoyens" du Commonwealth}

L'autre cas de figure, en Amérique du Nord et en Amérique centrale, n'est pas propre à cette région, mais est lié à une organisation politique interétatique issue de l'Empire colonial britannique : le Commonwealth. Cette communauté, qui réunit 53 pays indépendants ${ }^{22}$, n'est pas régie par une norme imposant l'octroi du droit de vote aux citoyens de cette organisation dans les États membres (au contraire de l'Union européenne). Mais un certain nombre d'États membres (Grande-Bretagne, Maurice et les petits pays américains et caribéens) reconnaissent ce droit de vote, s'inscrivant ainsi dans une coutume instaurée dans le cadre de l'Empire colonial britannique, qui distinguait différents types de citoyenneté, et notamment une citoyenneté du Commonwealth séparée de la nationalité britannique. En 1918, la loi britannique sur la représentation du peuple avait accordé le droit de vote à tous les sujets de l'Empire. Au milieu du XXe siècle, ce droit a été maintenu pour les Irlandais (et l'Irlande a fait de même en 1985 pour les Britanniques). Au Royaume-Uni, les ressortissants des États indépendants membres du Commonwealth continuent aujourd'hui de bénéficier de ce droit au même titre que les Irlandais. Cette situation découle de la persistance (formelle, voire fictive) d'une forme d'allégeance à la Reine. En fait, dans un certain sens, les "citoyens" du Commonwealth ne sont pas vraiment des étrangers, mais des sujets de la Couronne. C'est cette tradition qui persiste dans les États américains et caribéens membres

21. On trouvera des développements plus précis sur cette question dans ANDRÈS, Hervé, Le droit de vote des étrangers, op. cit., pp. 289 et ss.

22. Afrique du Sud, Antigua-et-Barbuda, Australie, Bahamas, Bangladesh, Barbade, Belize, Botswana, Brunei, Cameroun, Canada, Chypre, Dominique, Fidji, Gambie, Ghana, Grenade, Guyana, Inde, Jamaïque, Kenya, Kiribati, Lesotho, Malaisie, Malawi, Maldives, Malte, Maurice, Mozambique, Namibie, Nauru, Nigeria, Nouvelle-Zélande, Ouganda, Pakistan, Papouasie-Nouvelle-Guinée, Royaume-Uni, Saint-Christophe-et-Niévès, Saint-Vincent-et-les-Grenadines, Sainte-Lucie, Samoa îles Salomon, Seychelles, Sierra Leone, Singapour, Sri Lanka, Swaziland, Tanzanie, Tonga, Trinitéet-Tobago, Tuvalu, Vanuatu, Zambie. Voir http://www.thecommonwealth.org/Internal/142227/members/ 
du Commonwealth, qui accordent tous le droit de vote et d'éligibilité à tous les citoyens de cette organisation, pour tous les scrutins ${ }^{23}$.

Enfin, l'on peut noter que la Constitution de la République dominicaine prévoit la possibilité pour les étrangers résidant depuis plus de 10 ans sur son territoire d'exercer des fonctions électives dans les gouvernements locaux. Ainsi, à peu près la moitié des pays d'Amérique du Nord et d'Amérique centrale (12 sur 23 au total) reconnaît le droit de vote à des étrangers.

\section{En Amérique du Sud, une pratique généralisée}

Les États d'Amérique du Sud accordent en général le droit de vote aux résidents étrangers, et pas seulement lors de certaines élections locales. Seuls deux États (sur 12) réservent le droit de vote à leurs seuls nationaux (Équateur et Surinam). La règle générale est donc l'ouverture du droit de vote. Cela s'explique sans doute par l'histoire marquée par la colonisation et l'installation de nombreux migrants en provenance d'Europe. Ces pays sont des pays d'immigration, celle-ci étant assumée comme partie prenante de leur identité. Ainsi, les Constitutions actuelles portent encore la trace de ce caractère, en prévoyant des dispositions spécifiques favorisant l'installation et l'intégration des migrants. Parmi ces dispositions, le droit de vote des étrangers est souvent accordé explicitement dans les textes constitutionnels (Bolivie, Chili, Colombie, Guyana, Paraguay, Uruguay, Venezuela). En Argentine, certaines constitutions provinciales accordent le droit de vote lors de certaines élections aux étrangers résidant depuis un certain temps, pratique qui remonte au milieu du XIXe siècle ${ }^{24}$. Le Venezuela accorde le droit de vote lors des élections locales et intermédiaires (États) au bout de 10 ans de résidence. Le Brésil exclut le droit de vote pour les étrangers, sauf pour les Portugais, en vertu des liens coloniaux, d'une langue commune et d'un traité bilatéral de réciprocité. Le Guyana accorde le droit de vote aux citoyens du Commonwealth. Trois États accordent le droit de vote à tous les étrangers à toutes les élections (Chili, au bout de 5 ans de résidence, Uruguay au bout de 15 ans et Venezuela au bout de 10 ans).

23. II s'agit des 10 pays suivants : Antigua-et-Barbuda, Barbade, Belize, Dominique, Grenade, Jamaïque, Saint-Christophe-et-Niévès, Sainte-Lucie, Saint-Vincent-et-les Grenadines, Trinité-et-Tobago.

24. Cf. GONZÁLEZ BERNALDO, Pilar, "Una ciudadanía de residencia : la experiencia de los extranjeros en la ciudad de Buenos Aires (1882-1917)", Entrepasados, vol. XV, n 30, 2006. 
Il est à noter que cette ouverture vis-à-vis des droits politiques va de pair avec une ouverture du droit de la nationalité. La plupart des pays sud-américains pratiquent la naturalisation au bout d'un certain temps de résidence, la double nationalité (parfois sous condition de réciprocité) et le droit du sol (nationalité reconnue par le seul fait de naître sur le territoire de l'État).

Ainsi, comme cela a déjà été noté en Europe, il convient de rappeler que l'ouverture du droit de vote n'a aucun lien avec une fermeture présumée de la nationalité. Des pays avec une nationalité "ouverte" (facile à acquérir, que ce soit par le droit du sol ou par naturalisation) sont aussi des pays ayant ouvert leur droit de vote aux étrangers ${ }^{25}$.

\section{En Afrique, une pratique isolée, mais un vrai problème politique}

En Afrique, au moins huit pays sur 53 accordent le droit de vote à des étrangers. Il s'agit du Botswana, du Burkina Faso, du Cap-Vert, de la Guinée, de Maurice, de l'Ouganda, du Rwanda et de la Zambie. Le droit de vote est parfois limité aux ressortissants de certains pays (réciprocité, appartenance au Commonwealth), et parfois limité aux élections locales ${ }^{26}$. Une étude plus complète de la situation supposerait une confrontation entre les textes proclamés (Constitution, loi électorale...) et les pratiques réelles. La question du droit de vote n'a vraiment de sens que dans un cadre formellement démocratique, qui requiert certaines conditions politiques et sociales. Au-delà de la diversité des situations d'un bout à l'autre du continent, on peut affirmer globalement que les processus en cours depuis les années 90 (démocratisation électorale, instauration du multipartisme, libéralisation de l'économie, modification des flux migratoires) conduisent à poser la question du vote des étrangers de façon exacerbée, en des termes un peu différents des autres continents.

D'une part, il semble que le vote des étrangers a été pratiqué par plusieurs pays ${ }^{27}$, alors qu'un parti unique avait le monopole du

25. Cf. LE COUR GRANDMAISON, Olivier ; WIHTOL de WENDEN, Catherine (sous la direction de), Les étrangers dans la cité. Expériences européennes, Paris : Éd. La Découverte, 1993, 212 p.

26. Voir le détail dans ANDRÈS, Hervé, Le droit de vote des étrangers, op. cit.

27. Pour les cas de la Côte-d'Ivoire et de la Tanzanie, voir par exemple WHITAKER, Beth Elise, "Citizens and foreigners : democratization and the politics of exclusion in Africa", African Studies Review, vol. 48, n 1, April 2005, pp. 109-126. 
pouvoir et que la compétition électorale réelle était inexistante. Le vote des étrangers était utilisé pour augmenter formellement l'adhésion du peuple à son gouvernement. D'autre part, l'instauration du multipartisme a entraîné une réelle compétition pour le pouvoir, et de très nombreux gouvernants ${ }^{28}$ ont mis en doute la nationalité de leurs opposants pour leur interdire juridiquement l'accès aux élections. Cette stratégie politique a pour intérêt de limiter la compétition électorale en écartant des concurrents, de détourner l'attention des électeurs du bilan politique de ces gouvernements, et elle s'appuie sur une relative passivité des observateurs internationaux. Elle tend à contaminer l'ensemble du continent et à être la principale ressource rhétorique des différents partis luttant pour la conquête du pouvoir ou pour s'y maintenir.

En plaçant les questions de l'identité nationale et de la nationalité au centre du jeu politique, alors que la réalité des flux migratoires et les incertitudes sur les frontières héritées du passé colonial font que la catégorie "étranger" est sujette à toutes les interprétations arbitraires $^{29}$, cette stratégie a eu pour effet de contribuer à un développement important de la xénophobie. Dans le cas de la Côte-d'Ivoire, elle a conduit à un grave conflit militaire pouvant déboucher à terme sur un éclatement du pays. Ce cas est sûrement un des plus tragiques, car il tourne le dos à la tradition d'hospitalité, qui se traduisait notamment par la reconnaissance pendant une trentaine d'années du droit de vote des étrangers, en dépit de son caractère anticonstitutionnel. II s'agissait alors d'une pratique certes instrumentalisée par le pouvoir, certes discrétionnaire et discriminatoire (seuls les ressortissants d'Afrique subsaharienne en bénéficiaient) ${ }^{30}$, mais son retrait au milieu des années 90 a été un des éléments déclencheurs du processus dramatique du début du XXle siècle ${ }^{31}$. Dans de très nombreux pays, la question du vote des étrangers est utilisée aujourd'hui comme repoussoir par les partis d'opposition ${ }^{32}$, qui accusent le pouvoir de vouloir faire voter

28. On peut citer le Botswana, le Nigeria, la Zambie, la Côte-d'Ivoire, la Tanzanie, etc. Ibidem.

29. Ceci est d'autant plus vrai que dans de nombreux pays les règles appliquées conduisent à exiger comme condition d'éligibilité à la présidence de la République non seulement la possession de la nationalité, mais en outre d'être né de parents possédant cette même nationalité, ce qui est encore plus difficile à établir (et facile à contester) car cela renvoie en général aux périodes antérieures à la décolonisation.

30. Voir KOBO, Pierre-Claver, "Côte-d'Ivoire : les étrangers votent", Plein Droit, n 9, décembre 1989 , pp. 39-41.

31. Cf. BREDELOUP, Sylvie, "La Côte-d'Ivoire ou l'étrange destin de l'étranger", Revue Européenne des Migrations Internationales, vol. 19, $\mathrm{n}^{\circ}$ 2, 2003, pp. 85-111.

32. Par ailleurs, une autre question est posée dans de très nombreux pays : celle du droit de vote des expatriés. 
des "étrangers", qui peuvent être, selon les cas, des électeurs importés des pays voisins le jour même de l'élection, mais aussi des résidents de longue date, des réfugiés politiques ou des migrants économiques, ou encore des membres de minorités ethniques discriminées dont la nationalité est remise en cause. L'instrumentalisation de cette question dans le jeu politique fait courir le risque de multiples conflits. Se présentant comme l'affirmation des principes démocratiques modernes (en fait, du principe de souveraineté nationale), elle simplifie outrancièrement la question des identités politiques et contribue à affaiblir la démocratie en écartant de nombreuses personnes de la vie politique de leur pays de résidence, et donc en portant atteinte aux principes d'égalité et de légitimation des gouvernements.

Dans l'exemple africain, on peut établir le lien consubstantiel entre la question du vote des étrangers et celle de la démocratie. Hors du système démocratique, la question ne se pose même pas. Mais par ailleurs, la nationalité, contrairement à l'interprétation qui en fait l'idéal type de l'intégration politique ${ }^{33}$, apparaît bien dans cet exemple comme une ressource importante pour l'exclusion politique. En Afrique, peut-être encore plus qu'ailleurs, la nationalité sert d'abord à exclure du jeu politique.

\section{En Asie, une pratique rare, mais en débat dans certains pays}

En Asie, au moins deux États (Israël et la Corée du Sud) et un territoire (Hong-Kong) pratiquent le droit de vote des étrangers.

Sur le territoire annexé de Jérusalem-Est, Israël accorde le droit de vote au niveau local (mais pas à la Knesset) aux "étrangers" résidents permanents - y compris à de nombreux Palestiniens - qui n'ont pas la nationalité israélienne. Cette situation est héritée du processus historique par lequel Israël a privé la population résidente de la citoyenneté.

La Corée du Sud a accordé récemment le droit de vote lors des élections municipales aux étrangers ayant un statut de résident permanent depuis plus de trois ans, statut difficile à obtenir car les conditions sont extrêmement restrictives. D'après les chiffres officiels, moins de

33. Cf. SCHNAPPER, Dominique, La communauté des citoyens. Sur lidée moderne de nation,

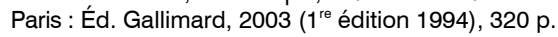


7000 étrangers (dont $99 \%$ de Taïwanais) ont pu voter en mai 2006 pour la première fois, soit un pourcentage très réduit de la population étrangère.

Cette ouverture coréenne s'explique sans doute en partie par la volonté d'influer sur le débat en cours au Japon, en faisant preuve de bonne volonté et en espérant que le Japon appliquera le principe de réciprocité. II faut dire que cette question fait régulièrement partie des discussions bilatérales entre les deux pays, la Corée du Sud réclamant avec constance l'instauration du droit de vote au niveau local pour les Coréens résidant au Japon.

Au Japon, la question du droit de vote des étrangers est débattue depuis de nombreuses années ${ }^{34}$, notamment parce qu'elle concerne un demi-million de Coréens nés au Japon et dont les familles y sont établies depuis plusieurs générations. À l'origine, il s'agit de Coréens colonisés par l'Empire japonais, "importés" au Japon, puis privés de leur nationalité japonaise après la Deuxième Guerre mondiale ${ }^{35}$. Ils y bénéficient d'un statut de résident permanent, mais ne peuvent pas accéder à la nationalité japonaise. Dans les années 90 , la Cour suprême a considéré comme anticonstitutionnelle la proposition d'accorder le droit de vote aux étrangers, même résidents de longue date. L'Assemblée de la métropole de Tokyo a créé en 1997 un Conseil consultatif des étrangers. En 2000, une proposition de loi reconnaissant le droit de vote au niveau local aux seuls Coréens résidents permanents, soutenue par une partie de la majorité parlementaire et de l'opposition, a été repoussée. La question reste en débat depuis lors.

Ce débat s'inscrit dans le cadre général des luttes menées contre le racisme, pour le respect des droits des immigrés au Japon ${ }^{36}$, et aussi dans le cadre des relations postcoloniales entre l'ancienne puissance impériale et ses satellites ayant conquis leur souveraineté ${ }^{37}$.

À Hong-Kong, ancienne colonie britannique rattachée à la République populaire de Chine en 1997 avec un statut de région adminis-

34. Cf. NAKANO, Yuji, "Les propositions de loi sur le droit de vote des étrangers aux élections locales au Japon", Migrations Société, vol. 14, n 83, septembre-octobre 2002, pp. 9-25.

35. De nombreux Chinois de Taïwan sont dans le même cas.

36. Ces luttes concernent également d'autres minorités ethniques. Voir YAMANAKA, Keiko, "A breakthrough for ethnic minority rights in Japan. Ana Bortz's courageous challenge", in : MOROKVASIC-MÜLLER, Mirjana ; EREL, Umut ; SHINOZAKI, Kyoko (Eds.), Crossing borders and shifting boundaries, Opladen : Leske \& Budrich, 2003, pp. 231-259.

37. Cf. AHN, Deok Keun Matthew, "Reflections on voting, identity, and self-affirmation in Japan", Harvard Asia Quarterly, vol. 4, n 4, Autumn 2000, http://www.asiaquaterly.com/content/view/84/40 
trative spéciale, les résidents permanents (qui sont les personnes de toutes nationalités bénéficiant du droit de résider de façon permanente à Hong-Kong) ont le droit de vote à toutes les élections.

\section{En Océanie, une pratique liée à l'immigration européenne}

En Australie, le droit de vote est accordé pour toutes les élections et référendums aux British Subjects, qui sont les ressortissants des 48 États membres du Commonwealth en 1984 ou considérés comme tels, comme l'Irlande. Le nombre de ces électeurs non australiens tend à se réduire au fil des années. En outre, trois États australiens (sur huit) reconnaissent le droit de vote aux résidents étrangers pour les élections locales (Australie-Méridionale, Tasmanie et Victoria). Par ailleurs, on peut signaler que l'lle de Norfolk reconnaît le droit de vote et d'éligibilité pour l'élection à l'Assemblée législative et le droit de vote aux référendums à tous les résidents, quelle que soit leur nationalité. Un délai minimum de résidence de 2 ans et 5 mois est requis. Le gouvernement fédéral propose que le vote des étrangers soit supprimé et que le délai de résidence (pour les Australiens) soit réduit. Le conflit qui oppose le gouvernement de l'île et son Assemblée au gouvernement central dure depuis plusieurs années.

En Nouvelle-Zélande, tout résident permanent, quelle que soit sa nationalité, a le droit de vote à tous les scrutins, mais le droit d'éligibilité est réservée aux Néo-Zélandais.

À notre connaissance, les autres États océaniens n'accordent pas le droit de vote aux étrangers, sauf les îles Cook (État non membre de l'ONU), qui reconnaissent ce droit aux citoyens du Commonwealth et aux titulaires d'un statut de résident permanent.

\section{En synthèse : une pratique loin d'être anecdotique}

Ainsi, actuellement, dans au moins un pays sur trois dans le monde (65 États sur 192 pays membres de l'ONU), des étrangers peuvent voter à des élections politiques. Ces chiffres étant minimaux, compte tenu par ailleurs du nombre de pays où l'on est très loin des "normes"38 démocratiques, l'on peut affirmer que le droit de vote des étrangers n'est absolument pas une utopie, une aberration, une exception euro-

38. Ces normes sont d'ailleurs très discutables... 
péenne. La profondeur historique du vote des étrangers aux ÉtatsUnis et ailleurs, la diversité des situations historiques et politiques ainsi que l'existence du vote d'étrangers sur tous les continents conduisent à ébranler le paradigme selon lequel le vote serait "naturellement" une prérogative de la nationalité.

L'ouverture du droit de vote est réelle. Si l'on considère, par continent, le nombre d'États ouvrant (au moins de façon limitée) le droit de vote à certains étrangers, on peut dresser le tableau suivant.

Tableau1 : Répartition par continent des pays "ouverts" au droit de vote des étrangers

\begin{tabular}{|l|c|c|}
\hline Continent ou région & Nombre de pays & Nombre de pays "ouverts"40 \\
\hline Europe & 44 & $30^{41}$ \\
\hline $\begin{array}{l}\text { Amérique du Nord et } \\
\text { Amérique centrale }\end{array}$ & 23 & 12 \\
\hline Amérique du Sud & 12 & 10 \\
\hline Afrique & 53 & 8 \\
\hline Asie & 46 & 3 \\
\hline Océanie & 14 & 2 \\
\hline Total & 192 & 65 (minimum) $)^{42}$ \\
\hline
\end{tabular}

Cette ouverture ne se fait pas, concrètement, par un passage du "tout fermé" au "tout ouvert". En fait, il y a deux principales modalités d'ouverture possible : l'une porte sur les échelons du vote et l'autre porte sur les ayants droit de cette ouverture.

Certains pays accordent ainsi le droit de vote aux étrangers, mais seulement pour certains types de scrutins, par exemple les scrutins locaux.

39. Membres de l'ONU.

40. Nous entendons par pays "ouvert" tout pays ayant accordé le droit de vote au moins sur une partie de son territoire, ou pour certains types d'élections, à certaines catégories d'étrangers.

41. Ces 30 pays sont les 27 pays membres de l'Union européenne (qui ont ouvert au minimum le droit de vote lors des élections municipales et européennes aux "citoyens de l'Union"), la Suisse (plusieurs cantons accordent le droit de vote lors des élections communales, voire cantonales, parfois depuis très longtemps : Neuchâtel en 1849), la Norvège (1979 pour le vote des citoyens des pays du Conseil nordique, 1983 pour les autres) et l'Islande (1986 pour le Conseil nordique, 2002 pour les autres).

42. On trouve le détail des données présentées ici dans ANDRÈS, Hervé, Le droit de vote des étrangers, op. cit. On a intégré depuis le cas de la Roumanie, dont l'entrée dans l'Union européenne en 2007 a entraîné l'ouverture du droit de vote lors des élections municipales et européennes prévu par les traités européens. 
L'autre modalité d'ouverture porte sur la qualité des étrangers auxquels le droit de vote est accordé. Ainsi, certains pays octroient ce droit seulement aux ressortissants de pays avec lesquels ils sont liés soit par des accords de réciprocité (par exemple l'Union européenne), soit par des liens postcoloniaux (Commonwealth).

L'ouverture est réalisée selon ces modalités, souvent combinées.

Tableau 2 : Modalités d'ouverture du droit de vote aux étrangers

\begin{tabular}{|c|c|c|c|c|c|c|c|c|}
\hline & & Europe & $\begin{array}{l}\text { Amérique } \\
\text { du Nord } \\
\text { et } \\
\text { Amérique } \\
\text { centrale }\end{array}$ & $\begin{array}{c}\text { Amérique } \\
\text { du Sud }\end{array}$ & Afrique & Asie & Océanie & Total \\
\hline Nombre de pay & & 44 & 23 & 12 & 53 & 46 & 14 & 192 \\
\hline & $\begin{array}{l}\text { ayant une pratique } \\
\text { du droit de vote des } \\
\text { étrangers, spécifique } \\
\text { selon certaines natio- } \\
\text { nalités }\end{array}$ & 28 & 10 & 2 & 3 & 0 & 1 & 44 \\
\hline $\begin{array}{l}\text { Pays "ouverts" } \\
\text { (ne restreignant }\end{array}$ & $\begin{array}{l}\text { ayant une pratique } \\
\text { d'ouverture du droit } \\
\text { de vote pour tous } \\
\text { les étrangers }\end{array}$ & 16 & 2 & 8 & 5 & 3 & 2 & 36 \\
\hline $\begin{array}{l}\text { pas strictement } \\
\text { le droit de vote } \\
\text { à leurs seuls }\end{array}$ & \begin{tabular}{|l|} 
ayant une pratique \\
d'ouverture \\
(total) 43
\end{tabular} & 30 & 12 & 10 & 8 & 3 & 2 & 65 \\
\hline ressortissants) & $\begin{array}{l}\text { pratiquant le droit } \\
\text { de vote des } \\
\text { étrangers au niveau } \\
\text { national }\end{array}$ & 4 & 10 & 4 & 3 & 1 & 2 & 24 \\
\hline & $\begin{array}{l}\text { pratiquant le droit } \\
\text { de vote des } \\
\text { étrangers au niveau } \\
\text { local }\end{array}$ & 30 & 12 & 10 & 8 & 3 & 2 & 65 \\
\hline $\begin{array}{l}\text { Pays "fermés" (re } \\
\text { le droit de vote à } \\
\text { tissants, ou pour } \\
\text { de données et qu } \\
\text { pas accorder le c } \\
\text { étrangers). }\end{array}$ & $\begin{array}{l}\text { estreignant strictement } \\
\text { leurs seuls ressor- } \\
\text { lesquels l'on n'a pas } \\
\text { 'on a présumés ne } \\
\text { droit de vote à des }\end{array}$ & 14 & 11 & 2 & 45 & 43 & 12 & 127 \\
\hline
\end{tabular}

43. Les chiffres de cette ligne ne correspondent pas à la somme des deux lignes précédentes car l'ouverture peut combiner les deux critères : ouverture de certains scrutins à tous les étrangers, ouverture d'autres scrutins à certaines catégories d'étrangers. 
Sur les 65 pays pratiquant le droit de vote des étrangers, plus de la moitié (36) le font en direction de tous les ressortissants étrangers, quelle que soit leur nationalité.

L'ouverture est toujours réalisée au moins au niveau local. Mais de nombreux États ouvrent également le droit de vote lors des scrutins intermédiaires (élections "régionales", "cantonales" ou des entités fédérées). Et 24 États ouvrent également le droit de vote lors des scrutins au niveau national. Parfois, ce sont seulement une partie des scrutins nationaux qui sont ouverts (référendums en Suède par exemple), mais pas la totalité.

Les 24 États qui accordent un droit de vote au niveau national le réservent dans la majorité des cas à certaines nationalités sélectionnées en fonction des liens postcoloniaux ou du principe de réciprocité. Mais dans cinq pays (dont Hong-Kong ${ }^{44}$ ), le droit de vote au niveau national est accordé à tous les résidents remplissant certaines conditions.

Tableau 3 : Modalités d'ouverture pour le droit de vote au niveau national

\begin{tabular}{|c|c|c|c|}
\hline $\begin{array}{l}\text { Droit de vote au } \\
\text { niveau national }\end{array}$ & $\begin{array}{c}\text { Droit de vote réservé à certaines } \\
\text { nationalités }\end{array}$ & $\begin{array}{l}\text { Droit de vote accordé à } \\
\text { toutes les nationalités }\end{array}$ & Total \\
\hline Europe & $\begin{array}{l}\text { Irlande (Britanniques et réciprocité) } \\
\text { Royaume-Uni (Irlandais et citoyens } \\
\text { du Commonwealth) } \\
\text { Portugal (Brésiliens) }\end{array}$ & $\begin{array}{l}\text { Suède : seulement aux } \\
\text { référendums }\end{array}$ & 4 \\
\hline $\begin{array}{l}\text { Amérique du Nord } \\
\text { et Amérique } \\
\text { centrale }\end{array}$ & $\begin{array}{ll}\text { Pays du Commonwealth pour les } & 10 \\
\text { citoyens du Commonwealth }\end{array}$ & 0 & 10 \\
\hline Amérique du Sud & $\begin{array}{l}\text { Brésil (Portugais) } \\
\text { Guyana (citoyens du Commonwealth) }\end{array}$ & $\begin{array}{l}\text { Chili } \\
\text { Uruguay }\end{array}$ & 4 \\
\hline Afrique & $\begin{array}{l}\text { Botswana (liste définie par le } \\
\text { Parlement) } \\
\text { Guinée (réciprocité) } \\
\text { Maurice (citoyens du Commonwealth) }\end{array}$ & 0 & 3 \\
\hline Asie & & $\begin{array}{l}\text { Hong-Kong (territoire } \\
\text { Rép. populaire de Chine) }\end{array}$ & 1 \\
\hline Océanie & Australie (British Subjects) & Nouvelle-Zélande & 2 \\
\hline Total & 19 & 5 & 24 \\
\hline
\end{tabular}

44. Région administrative à statut spécial de la République populaire de Chine, depuis 1997. 
Une troisième modalité d'ouverture mériterait sans doute d'être citée, qui joue un rôle notamment dans au moins trois États fédéraux : il s'agit des dispositions qui permettent aux collectivités fédérées, voire aux communes elles-mêmes, d'accorder le droit de vote aux étrangers (cas de la Suisse, des États-Unis ou de l'Australie). En effet, la détermination du droit de vote ne relève pas toujours forcément de l'échelon central (de l'État souverain) et, au moins pour des scrutins locaux, des collectivités locales peuvent déterminer elles-mêmes qui a le droit de vote.

L'on observera que la complexité du tableau de la situation actuelle quant au droit de vote des étrangers remet en question une solution pratique souvent évoquée dans le débat : celle de la réciprocité. lgnorant la réalité du droit de vote des étrangers dans plus de 60 pays dans le monde, certains proposent d'appliquer, au même titre que ce qui a été fait pour l'Union européenne, la norme de la réciprocité. Seraient bénéficiaires, en France, du droit de vote, les étrangers ressortissants de pays accordant le droit de vote aux Français. Mais comment actualiser, en permanence, la liste de ces pays? Comment traiter les Suisses dans leur diversité, vu que certains cantons, voire certaines communes accordent le droit de vote aux Français? Faudrait-il en passer par un accord de réciprocité expresse, comme c'est le cas en Espagne? L'expérience montre que cela est difficile à mettre en œuvre et à actualiser. Ceci dit, la multiplication des exemples d'ouverture du droit de vote pourrait bien entraîner, dans une sorte de cercle vertueux de réciprocité globale, une multitude d'États à évolver, pour prendre en compte de façon pratique le décalage entre la population constitutive et la population résidente.

Si l'on pouvait introduire dans ce schéma statique mis à jour mi2006 une dimension dynamique intégrant les évolutions en cours, on observerait probablement un mouvement clair dans la direction de la reconnaissance du vote des étrangers. Certains pays le pratiquent depuis longtemps, mais la plupart des États ont ouvert le droit de vote à la fin du $X X X^{e}$ siècle ou au début du XXle. Malgré l'existence de crispations nationalistes ici (en Europe, par exemple) ou là (en Afrique), il semble bien se dessiner une tendance vers l'ouverture. II est avéré que l'on ne peut considérer comme définitive la règle de l'exclusion des étrangers du droit de vote. Le mouvement d'ouverture touche à ce jour au moins un pays sur trois. On peut légitimement penser que ce mouvement se poursuivra, et que la règle d'exclusion, évoquée au début de cette contribution, est considérablement ébranlée. 
